\title{
Validation of evaluated neutron standard cross sections
}

\author{
S. Badikov a and T. Golashvili ${ }^{\mathrm{a}}$ \\ Central Research Institute of Management, Economics and Information (Atominform), 127434 Moscow, Russia
}

\begin{abstract}
Some steps of the validation and verification of the new version of the evaluated neutron standard cross sections were carried out. In particular, 1) the evaluated covariance data was checked for physical consistency, 2) energy-dependent evaluated cross-sections were tested in most important neutron benchmark field $-{ }^{252} \mathrm{Cf}$ spontaneous fission neutron field, 3) a procedure of folding differential standard neutron data in group representation for preparation of specialized libraries of the neutron standards was verified. The results of the validation and verification of the neutron standards can be summarized as follows: a) the covariance data of the evaluated neutron standards is physically consistent since all the covariance matrices of the evaluated cross sections are positive definite, b) the ${ }^{252} \mathrm{Cf}$ spectrum averaged standard cross-sections are in agreement with the evaluated integral data (except for ${ }^{197} \mathrm{Au}(\mathrm{n}, \gamma)$ reaction), $\left.\mathrm{c}\right)$ a procedure of folding differential standard neutron data in group representation was tested, as a result a specialized library of neutron standards in the ABBN 28-group structure was prepared.
\end{abstract}

\section{Introduction}

The procedure of neutron cross section evaluation consists of few steps: 1) compilation of an experimental data base and critical review of the results of measurements (including rejection of evidently wrong data, correction and renormalization of the experimental data to new values of decay constants and standards), 2) statistical analysis of the accepted experimental data for getting evaluated values and their covariances, 3) validation and verification of the evaluated data (comparison of the evaluated values with results of integral measurements in neutron benchmark fields and critical assemblies, testing the covariance data for physical consistency - the covariance matrices of uncertainties of the evaluated cross-sections must be positive definite).

A new version of neutron standard cross sections was released in October 2005. In accordance with the final step of the evaluation procedure new neutron standards must be subject to a process of validation and verification. This paper is devoted to the description of results of the validation and verification of the neutron standards. In particular, the results of checking the evaluated standard covariance data for positive definiteness are presented in section 2. Comparison of evaluated values with results of cross section measurements in ${ }^{252} \mathrm{Cf}$ spontaneous neutron fission field is carried out in section 3 . In addition, the evaluated data must be transformed in group structure for use in applications. Section 4 describes an algorithm and the results of the transformation of the neutron standards in $\mathrm{ABBN}$ group representation.

This study includes information on neutron standard cross sections for 8 reactions: ${ }^{6} \mathrm{Li}(\mathrm{n}, \mathrm{t}),{ }^{10} \mathrm{~B}\left(\mathrm{n}, \alpha_{0}\right),{ }^{10} \mathrm{~B}\left(\mathrm{n}, \alpha_{1}\right)$, ${ }^{197} \mathrm{Au}(\mathrm{n}, \gamma),{ }^{235} \mathrm{U}(\mathrm{n}, \mathrm{f}),{ }^{238} \mathrm{U}(\mathrm{n}, \mathrm{f}),{ }^{238} \mathrm{U}(\mathrm{n}, \gamma),{ }^{239} \mathrm{Pu}(\mathrm{n}, \mathrm{f})$.

\section{Test of covariance matrices for positive definiteness}

The covariance matrices of experimental and estimated errors must be positive definite. This requirement can be justified in

\footnotetext{
${ }^{a}$ e-mail: badikov@ainf.ru, gol@ainf.ru
}

the following way. At first order a variance of any scalar function from a random vector is a quadratic form. The covariance matrix of the random vector is the matrix of the quadratic form. So, the variance of any function will be positive only in the case of positive definiteness of the covariance matrix.

Covariance matrices of experimental and estimated data are widely used in the statistical analysis of measurements. Since the correlations between experimental errors can not be measured, both covariance matrices of experimental and estimated errors include calculated values. For this reason calculated covariance matrices must be tested for positive definiteness at each step of the evaluation procedure.

The evaluation of neutron cross section standards was carried out on the basis of generalized least squares method. The method of the evaluation was described in detail in papers $[1,2]$. The evaluation consisted in calculation of relative corrections $\delta \vec{\sigma}$ to former neutron cross section standards induced by new measurements:

$$
\begin{gathered}
\delta \vec{\sigma}=\left[\mathbf{X}^{\mathrm{T}} \mathbf{V}^{-1} \mathbf{X}\right]^{-1} \mathbf{X}^{\mathrm{T}} \mathbf{V}^{-1} \delta \vec{y} \\
\mathbf{W}=\left[\mathbf{X}^{\mathrm{T}} \mathbf{V}^{-1} \mathbf{X}\right]^{-1} .
\end{gathered}
$$

In (1) and (2) $\delta \vec{y}$ and $\delta \vec{\sigma}$ - vectors of deviations of $N$ new measurements from former evaluation and $L$ estimated corrections (parameters), $N>L, \mathbf{V}$ and $\mathbf{W}$ - covariance matrices of the deviations and estimated parameters correspondingly, $\mathbf{X}$ - a matrix of sensitivity coefficients. Preservation of positive definiteness in expressions of type (2) was considered in [3]. As follows from conclusions presented in [3] the matrix $\mathbf{W}$ is positive definite if the matrix $\mathbf{V}$ is positive definite and rank $\mathbf{X}=L$. In the calculations the orders of magnitude of $L$ and $N$ values are $\sim 10^{3}$ and $\sim 10^{4}$. As a consequence, testing the matrix $\mathbf{W}$ for positive definiteness by means of checking the matrix $\mathbf{V}$ and calculation of the rank of the matrix $\mathbf{X}$ are impossible due to restrictions in computer memory. For this reason, the only way of testing is checking the sub-matrices forming main diagonal of the matrix $\mathbf{W}$. Each of these sub-matrices corresponds to a covariance matrix of the uncertainties of some standard cross section. It should be noted that testing these sub-matrices can be carried out after their formatting in 
Table 1. Maximum and minimum eigenvalues of covariance matrices (as given in file 33) of uncertainties of the standard cross sections.

\begin{tabular}{lcc}
\hline reaction & $\max$ & $\min$ \\
\hline${ }^{6} \mathrm{Li}(\mathrm{n}, \mathrm{t})$ & $0.18506-2$ & $0.15987-6$ \\
${ }^{10} \mathrm{~B}\left(\mathrm{n}, \alpha_{0}\right)$ & $0.21079-1$ & $0.55488-6$ \\
${ }^{10} \mathrm{~B}\left(\mathrm{n}, \alpha_{1}\right)$ & $0.30782-2$ & $0.62965-7$ \\
${ }^{197} \mathrm{Au}(\mathrm{n}, \gamma)$ & $0.32202-1$ & $0.19718-5$ \\
${ }^{235} \mathrm{U}(\mathrm{n}, \mathrm{f})$ & $0.16139-1$ & $0.25738-5$ \\
${ }^{238} \mathrm{U}(\mathrm{n}, \mathrm{f})$ & $0.16741-1$ & $0.21347-4$ \\
${ }^{238} \mathrm{U}(\mathrm{n}, \gamma)$ & $0.42291-2$ & $0.20244-4$ \\
${ }^{239} \mathrm{Pu}(\mathrm{n}, \mathrm{f})$ & $0.16536-1$ & $0.49284-5$ \\
\hline
\end{tabular}

file 33 in correspondence with the prescriptions of the ENDF system.

The results of testing relative covariance matrices of uncertainties of evaluated standard cross sections (presented in file 33) for positive definiteness are given in table 1 where the maximum and minimum eigenvalues of the covariance matrices are listed. As follows from table 1 all the covariance matrices are positive definite. The eigenvalues of the matrices are concentrated at relatively narrow numerical intervals the eigenvalues change by 4-5 orders of magnitude. Thus, from the point of view of positive definiteness the evaluated standard covariance data is physically consistent.

\section{Validation of neutron standards with integral data}

Testing the energy-dependent differential evaluated data in neutron benchmark fields is a necessary step of the evaluation procedure. Such testing may detect troubles in the evaluated data in energy ranges where cross-section is most sensitive to results of integral measurements. Of all the neutron benchmark fields the ${ }^{252} \mathrm{Cf}$ neutron spontaneous fission spectrum is of special importance since 1) it is known with relatively high accuracy [4], 2) it has a rather simple structure, 3) small uncertainties of integral measurements (of the order of $2 \%$ ) can be achieved.

First we will derive formula for average (over neutron spectrum) cross-section $\bar{\sigma}$ and its uncertainty $\delta \bar{\sigma}$. In accordance with definition

$$
\bar{\sigma}=\int_{E_{\min }}^{E_{\max }} \sigma(E, \vec{\theta}) \varphi(E, \vec{\mu}) d E / \int_{E_{\min }}^{E_{\max }} \varphi(E, \vec{\mu}) d E .
$$

Here $\sigma(E, \vec{\theta})$ - a differential cross-section, $\varphi(E, \vec{\mu})-$ neutron spectrum, $\vec{\theta}$ and $\vec{\mu}$ - vectors of parameters with known covariance matrices $\operatorname{Var}(\vec{\theta})$ and $\operatorname{Var}(\vec{\mu})$. If the spectrum $\varphi(E, \vec{\mu})$ is given in a form of stepwise function

$$
\varphi(E, \vec{\mu})=\mu_{i}=\varphi_{i} \quad \text { if } \quad E_{i} \leq E<E_{i+1},
$$

Table 2. Calculated ${ }^{252} \mathrm{Cf}$ spectrum averaged neutron standard cross sections in comparison with evaluated integral data [5].

\begin{tabular}{lccccc}
\hline reaction & $\begin{array}{c}\text { energy } \\
\text { range, } \mathrm{MeV}\end{array}$ & $\begin{array}{c}\bar{\sigma}(\text { calc }) \\
\mathrm{b}\end{array}$ & $\begin{array}{c}\Delta \bar{\sigma}(\mathrm{calc}) \\
\%\end{array}$ & $\begin{array}{c}\bar{\sigma}(\mathrm{ev}) \\
\mathrm{b}\end{array}$ & $\begin{array}{c}\Delta \bar{\sigma} \\
(\mathrm{ev}), \%\end{array}$ \\
\hline${ }^{6} \mathrm{Li}(\mathrm{n}, \mathrm{t})$ & $0.015-2.80$ & 0.284 & 0.823 & - & - \\
${ }^{10} \mathrm{~B}\left(\mathrm{n}, \alpha_{0}\right)$ & $0.015-1.0$ & 0.0408 & 2.45 & - & - \\
${ }^{10} \mathrm{~B}\left(\mathrm{n}, \alpha_{1}\right)$ & $0.015-1.0$ & 1.852 & 1.42 & - & - \\
${ }^{197} \mathrm{Au}(\mathrm{n}, \gamma)$ & $0.015-2.80$ & 0.0698 & 0.810 & 0.0768 & 1.59 \\
${ }^{235} \mathrm{U}(\mathrm{n}, \mathrm{f})$ & $0.015-20.0$ & 1.223 & 0.293 & 1.210 & 1.2 \\
${ }^{238} \mathrm{U}(\mathrm{n}, \mathrm{f})$ & $1.00-20.0$ & 0.317 & 0.407 & 0.326 & 1.64 \\
${ }^{238} \mathrm{U}(\mathrm{n}, \gamma)$ & $0.015-2.20$ & 0.0614 & 1.035 & - & - \\
${ }^{239} \mathrm{Pu}(\mathrm{n}, \mathrm{f})$ & $0.015-20.0$ & 1.796 & 0.354 & 1.812 & 1.37 \\
\hline
\end{tabular}

formula (3) can be transformed in the following way

$$
\bar{\sigma}=\sum_{i=1}^{n} \frac{\varphi_{i}\left(E_{i+1}-E_{i}\right)^{\prime}}{\int_{E_{\min }}^{E_{\max }} \varphi(E, \vec{\mu}) d E} \frac{\int_{E_{i}}^{E_{i+1}} \sigma(E, \vec{\theta}) d E}{E_{i+1}-E_{i}}=\sum_{i=1}^{n} F_{i} \bar{\sigma}_{i}{ }^{\prime} .
$$

At first order random deviation $\delta \bar{\sigma}$ in the average cross section induced by the deviation in parameters is as follows

$$
\delta \bar{\sigma}=\sum_{k=1}^{L} \frac{\partial \bar{\sigma}}{\partial \theta_{k}} \delta \theta_{k}+\sum_{j=1}^{n} \frac{\partial \bar{\sigma}}{\partial F_{j}} \delta F_{j}
$$

Then,

$$
\operatorname{Var}(\bar{\sigma})=\left(\frac{\partial \bar{\sigma}}{\partial \vec{\theta}}\right)^{T} \operatorname{Var}(\vec{\theta})\left(\frac{\partial \bar{\sigma}}{\partial \vec{\theta}}\right)+\left(\frac{\partial \bar{\sigma}}{\partial \vec{F}}\right)^{T} \operatorname{Var}(\vec{F})\left(\frac{\partial \bar{\sigma}}{\partial \vec{F}}\right)
$$

where

$$
\frac{\partial \bar{\sigma}}{\partial \theta_{k}}=\sum_{i=1}^{n} F_{i} \frac{\int_{E_{i}}^{E_{i+1}} \frac{\partial \sigma(E, \vec{\theta})}{\partial \theta_{k}} d E}{E_{i+1}-E_{i}} \quad \frac{\partial \bar{\sigma}}{\partial F_{m}}=\bar{\sigma}_{m}=\frac{\int_{E_{m}}^{E_{m+1}} \sigma(E, \vec{\theta}) d E}{E_{m+1}-E_{m}} .
$$

Calculated ${ }^{252} \mathrm{Cf}$ spectrum averaged neutron standard crosssections are given in table 2 in comparison with integral data evaluated by W. Mannhart [5]. The differential evaluated cross sections were averaged over energy intervals (see column 2) where the cross-sections are used as neutron standards. The blanks in table 2 indicate the absence of integral evaluated data.

\section{Transformation of the neutron standard data in $A B B N$ group structure}

For use in reactor applications the evaluated neutron cross section data must be transformed in a group structure. The ABBN 28-group representation is widely used in reactor physics [6]. So, it would be useful to transform the evaluated neutron cross section standards in ABBN group structure.

Input information for the transformation is the data from the file 3 (cross sections) and file 33 (covariances) of the 
Table 3. Uncertainties and correlations of the standard ${ }^{238} \mathrm{U}(\mathrm{n}, \mathrm{f})$ reaction cross-section in $\mathrm{ABBN}$ group representation.

\begin{tabular}{llllllllllll}
\hline group & energy & \multicolumn{1}{l}{$\Delta \sigma}$, & & & & & \\
number & $\mathrm{MeV}$ & $\sigma, \mathrm{mb}$ & $\%$ & & & & & & \\
\hline 23 & 0.8 & 40.273 & 0.83 & 100 & & & & & \\
24 & 1.4 & 478.11 & 0.58 & 58 & 100 & & & & \\
25 & 2.5 & 539.75 & 0.58 & 49 & 72 & 100 & & & \\
26 & 4.0 & 587.40 & 0.60 & 46 & 65 & 76 & 100 & & \\
27 & 6.5 & 983.09 & 0.67 & 40 & 58 & 66 & 71 & 100 & \\
28 & 10.5 & 1055.8 & 0.62 & 38 & 55 & 61 & 61 & 64 & 100 \\
\hline
\end{tabular}

evaluation. The information in the file 33 is stored in a form of relative covariances of the average (over energy bins) evaluated cross sections:

$$
R_{l k}=\frac{\operatorname{cov}\left(\bar{\sigma}_{l}, \bar{\sigma}_{k}\right)}{\bar{\sigma}_{l} \bar{\sigma}_{k}}
$$

where $\bar{\sigma}_{l}, \bar{\sigma}_{k}$ - average evaluated cross-sections at energy bins $l$ and $k$. Usually group structure is more coarse than bin representation, and each energy group $i$ includes $n_{i}$ energy bins. Then average group cross section $\bar{\sigma}_{i}$ can be presented as a linear combination of $n_{i}$ average bin crosssection $\bar{\sigma}_{i l}$

$$
\bar{\sigma}_{i}=\frac{1}{\Delta E_{i}} \int_{E_{i}} \sigma(E) d E=\sum_{l} \frac{\Delta E_{i l}}{\Delta E_{i}} \bar{\sigma}_{i l} .
$$

Using the formula (7) results also in linear expansion for covariance between group cross-sections $\bar{\sigma}_{i}$ and $\bar{\sigma}_{j}$

$$
\operatorname{cov}\left(\bar{\sigma}_{i}, \bar{\sigma}_{j}\right)=\sum_{l} \frac{\Delta E_{i l} \bar{\sigma}_{i l}}{\Delta E_{i}} \sum_{k} \frac{\Delta E_{j k} \bar{\sigma}_{j k}}{\Delta E_{j}} R_{l k} .
$$

Formulae (7) and (8) were applied for transformation of differential neutron standard cross-sections in ABBN-28 group structure. As an example the group cross sections, their uncertainties and correlations for ${ }^{238} \mathrm{U}(\mathrm{n}, \mathrm{f})$ reaction are given in table 3 .

\section{Summary}

Some steps of the validation and verification of the new version of the evaluated neutron standard cross-sections were carried out at final stage of the evaluation procedure. The results of the validation and verification are presented below.

1. All the covariance matrices (as given in file 33) of evaluated neutron standard cross-sections are positive definite. So, the covariance data of the evaluated neutron standards is physically consistent.

2. The ${ }^{252} \mathrm{Cf}$ spectrum averaged standard cross sections were compared with evaluated integral data when it was possible. For neutron reactions on fissile isotopes averaged cross-sections are in agreement with the integral data within declared uncertainties. For ${ }^{197} \mathrm{Au}(\mathrm{n}, \gamma)$ reaction the averaged cross section is essentially lower then evaluated integral value. Additional studies are required for understanding the reason of this disagreement.

3. The evaluated neutron standard cross-sections were transformed in the ABBN 28-group structure for use in reactor applications. Thus, the procedure of folding differential standard neutron data in group representation was tested. This procedure can be applied for preparation of specialized libraries of neutron standards.

\section{References}

1. W.P. Poenitz, in Proceedings of the Conference on Nuclear Data Evaluation Methods and Procedures, Brookhaven, 1981, edited by B.A. Magurno, S. Pearlstein Brown (BNL-NCS-51363, 1981), p. 249.

2. V.G. Pronyaev et al., in Proceedings of International Conference on Nuclear Data for Science and Technology, Santa-Fe, USA, 2004, edited by R.C. Haight, M.B. Chadwick, T. Kawano, P. Talou, AIP Conf. Proc. 769, Part 1, 808 (Melville, New York, 2005).

3. S.A. Badikov, Atomnaya Energiya 84, 426 (1998).

4. W. Mannhart, International Atomic Energy Agency, Report IAEA-TECDOC-410 (1987), p. 158.

5. W. Mannhart, ibid., p. 413.

6. Report INDC(NDS)-448, 2003, IAEA. 